\title{
An expanded domain of fgf 3 expression in the hindbrain of zebrafish valentino mutants results in mis-patterning of the otic vesicle
}

\author{
Su-Jin Kwak, Bryan T. Phillips, Rebecca Heck and Bruce B. Riley* \\ Biology Department, Texas A\&M University, College Station, TX 77843-3258, USA \\ *Author for correspondence (e-mail: briley@mail.bio.tamu.edu) \\ Accepted 9 August 2002
}

\section{SUMMARY}

The valentino (val) mutation in zebrafish perturbs hindbrain patterning and, as a secondary consequence, also alters development of the inner ear. We have examined the relationship between these defects and expression of $f g f 3$ and $f g f 8$ in the hindbrain. The otic vesicle in $\mathrm{val} / \mathrm{val}$ mutants is smaller than normal, yet produces nearly twice the normal number of hair cells, and some hair cells are produced ectopically between the anterior and posterior maculae. Anterior markers pax 5 and $n k x 5.1$ are expressed in expanded domains that include the entire otic epithelium juxtaposed to the hindbrain, and the posterior marker $z p 23$ is not expressed. In the mutant hindbrain, expression of $f g f 8$ is normal, whereas the domain of $f g f 3$ expression expands to include rhombomere 4 through rhombomere $X$ (an aberrant segment that forms in lieu of rhombomeres 5 and 6). Depletion of $f g f 3$ by injection of antisense morpholino (fgf3-MO) suppresses the ear patterning defects in val/val embryos: Excess and ectopic hair cells are eliminated, expression of anterior otic markers is reduced or ablated, and $z p 23$ is expressed throughout the medial wall of the otic vesicle. By contrast, disruption of $f g f 8$ does not suppress the $\mathrm{val} / \mathrm{val}$ phenotype but instead interacts additively, indicating that these genes affect distinct developmental pathways. Thus, the inner ear defects observed in $\mathrm{val} / \mathrm{val}$ mutants appear to result from ectopic expression of $f g f 3$ in the hindbrain. These data also indicate that val normally represses $f g f 3$ expression in $\mathrm{r} 5$ and $\mathrm{r} 6$, an interpretation further supported by the effects of misexpressing val in wild-type embryos. This is in sharp contrast to the mouse, in which fgf 3 is normally expressed in $\mathrm{r} 5$ and $\mathrm{r} 6$ because of positive regulation by kreisler, the mouse ortholog of val. Implications for co-evolution of the hindbrain and inner ear are discussed.

Key words: Inner ear, Hair cell, FGF signaling, ace, Morpholino, val, kreisler, pax5, Zebrafish

\section{INTRODUCTION}

Development of the inner ear requires interactions with adjacent hindbrain tissue. Many studies have shown that the hindbrain can induce otic placodes in adjacent ectoderm (Stone, 1931; Yntema, 1933; Harrison, 1935; Waddington, 1937; Jacobsen, 1963; Gallagher et al., 1996; Woo and Fraser, 1998; Groves and Bronner-Fraser, 2000). Several of the relevant hindbrain signals have recently been identified (reviewed by Whitfield et al., 2002). In zebrafish, two members of the FGF family of signaling molecules, Fgf3 and Fgf8, are expressed in the anlagen of rhombomere 4 (r4) during late gastrulation, when induction of the otic placode begins (Reifers et al., 1998; Phillips et al., 2001; Maroon et al., 2002). At this time, pax8 is induced in the adjacent otic anlagen. Disruption of both $f g f 3$ and $f g f 8$ prevents induction of the otic placode, and conditions that expand the expression domains of these genes lead to production of supernumerary or ectopic otic vesicles (Phillips et al., 2001; Raible and Brand, 2001; Vendrell et al., 2001; Maroon et al., 2002). In addition, disruption or depletion of $F g f 3$ perturbs inner ear development in chick and mouse (Represa et al., 1991; Mansour et al., 1993), and misexpression of Fgf3 in chick is sufficient to induce ectopic otic vesicles
(Vendrell et al., 2000). It has also been shown that chick Fgf19, which is expressed in a pattern similar to that of Fgf3 (Mahmood et al., 1995), cooperates with the hindbrain factor Wnt8c to induce a range of otic placode markers in tissue culture (Ladher et al., 2000). Thus, multiple hindbrain factors are involved in otic placode induction, and FGF signaling plays an especially prominent role.

Much less is known about the role played by hindbrain signals in later stages of inner ear development. Experiments in chick embryos show that rotation of the early otic vesicle about the anteroposterior axis reorients gene expression patterns in a manner suggesting that proximity to the hindbrain influences differentiation of cells within the otic vesicle $(\mathrm{Wu}$ et al., 1998; Hutson et al., 1999). In zebrafish, Xenopus, chick and mouse embryos, Fgf3 continues to be expressed in the hindbrain after otic placode induction (Mahmood et al., 1995; Mahmood et al., 1996; McKay et al., 1996; Lombardo et al., 1998; Phillips et al., 2001). This raises the question of whether this factor also helps regulate subsequent development of the otic placode or otic vesicle.

Analysis of the valentino ( $\mathrm{val}$ ) mutant in zebrafish provides indirect evidence that hindbrain signals are necessary for normal development of the otic vesicle (Moens et al., 1996; 
Moens et al., 1998). val encodes a bZip transcription factor that is normally expressed in $\mathrm{r} 5$ and r6. val/val mutants produce an abnormal hindbrain in which the $r 5 / 6$ anlagen fails to differentiate properly and gives rise to a single abnormal segment, rX, which shows confused segmental identity. Although the val gene is not expressed in the inner ear, val/val mutants produce otic vesicles that are small and malformed. As otic induction appears to occur normally in val/val mutants (Mendonsa and Riley, 1999), we infer that altered hindbrain patterning perturbs signals required for later aspects of otic development. Mice homozygous for a mutation in the ortholologous gene, kreisler (Mafb - Mouse Genome Informatics), also show later defects in development of the otic vesicle (Deol, 1964; Cordes and Barsh, 1994). The inner ear defects in kreisler mutants are thought to result from insufficient expression of $F g f 3$ in the hindbrain (McKay et al., 1996). In contrast to zebrafish, mouse $F g f 3$ is initially expressed at moderate levels in the hindbrain from $\mathrm{r} 1$ through r6. As development proceeds, expression downregulates in the anterior hindbrain but upregulates in r4 (Mahmood et al., 1996). After formation of the otic placodes, Fgf3 expression also upregulates in $\mathrm{r} 5$ and $\mathrm{r} 6$. This upregulation fails to occur in kreisler mutants, possibly accounting for subsequent patterning defects in the inner ear (McKay et al., 1996).

To examine the relationship between hindbrain and otic vesicle development in zebrafish, we have examined patterning of these tissues in wild-type and val/val mutant embryos. We find that val/val mutants produce excess and ectopic hair cells at virtually any position in the epithelium juxtaposed to the hindbrain. Expression of the anterior otic markers $n k x 5.1$ ( $h m x 3$ - Zebrafish Information Network) and pax 5 is also seen ectopically throughout this region of the otic vesicle. Conversely, expression of the posterior marker zp23 (pou23 Zebrafish Information Network) is ablated in val/val embryos. Analysis of hindbrain patterning shows that $f g f 3$ is misexpressed in the $\mathrm{rX}$ region of val/val mutants. Disruption of $f g f 3$ function by injection of an antisense morpholino oligomer blocks formation of ectopic hair cells and suppresses AP patterning defects in the otic vesicle of val/val mutants. By contrast, $f g f 8$ is expressed normally in val/val embryos, and loss of $f g f 8$ does not suppress the inner ear defects caused by the val mutation. These data indicate that the expanded domain of $f g f 3$ plays a crucial role in the etiology of inner ear defects in val/val mutants and suggest that Fgf3 secreted by r4 normally specifies anterior fates, suppresses posterior fates and stimulates hair cell formation in the anterior of the otic vesicle.

\section{MATERIALS AND METHODS}

\section{Strains}

Wild-type zebrafish embryos were derived from the AB line (Eugene, $\mathrm{OR})$. Mutations used in this study were valentino ( al $^{\mathrm{b} 337}$ ) and acerebellar $\left(\right.$ ace $\left.e^{\text {ti282a }}\right)$. Both of mutations were induced with ENU and are thought to be functional null alleles (Moens et al., 1996; Moens et al., 1998; Brand et al., 1998). Embryos were developed at $28.5^{\circ} \mathrm{C}$ in water containing $0.008 \%$ Instant Ocean salts. Embryonic ages are expressed as hours post-fertilization (h).

\section{Identification of mutant embryos}

Live val/val homozygotes were reliably identified after $19 \mathrm{~h}$ by the small size and round shape of the otic vesicle. In addition, fixed val/val embryos stained for pax2.1, pax5 or zp23 showed characteristic changes in posterior hindbrain patterning. At earlier stages, val/val mutants were identified by loss of krox20 (egr2 - Zebrafish Information Network) staining in rhombomere 5 (Moens et al., 1996). Live acelace (fgf8/fgf8 - Zebrafish Information Network) mutants were readily identified after $24 \mathrm{~h}$ by the absence of a midbrainhindbrain border and enlarged optic tectum (Brand et al., 1996). In addition, ace/ace specimens that were fixed and stained for pax2.1 or pax 5 showed no staining in the midbrain-hindbrain border. At earlier stages $(14 \mathrm{~h})$, ace/ace mutants were identified by loss of $f g f 3$ expression in the midbrain-hindbrain border.

\section{Whole-mount immunofluorescent staining}

Embryos were fixed in MEMFA (0.1 M MOPS at 7.4, 2 mM EGTA, $1 \mathrm{mM} \mathrm{MgSO} 4,3.7 \%$ formaldehyde) and stained as previously described (Riley et al., 1999). Primary antibodies used in this study were: polyclonal antibody directed against mouse Pax2 (Berkeley Antibody Company, 1:100 dilution), which also recognizes zebrafish pax2.1 (Riley et al., 1999); Monoclonal antibody directed against acetylated tubulin (Sigma T-6793, 1:100), which binds hair cell kinocilia (Haddon and Lewis, 1996). Secondary antibodies were Alexa 546 goat anti-rabbit IgG (Molecular Probes A-11010, 1:50) or Alexa 488 goat anti-mouse IgG (Molecular Probes A-11001, 1:50).

\section{Whole-mount in situ hybridization}

Whole-mount in situ hybridization was performed as described (Stachel et al., 1993) using riboprobes for $f g f 3$ (Kiefer et al., 1996a), fgf8 (Reifers et al., 1998), dlA (Appel and Eisen, 1998; Haddon et al., 1998b), pax5 (Pfeffer et al., 1998), dlx3 and msxc (Ekker et al., 1992), nkx5.1 (Adamska et al., 2000), otxl (Li et al., 1994), and zp23 (Hauptmann and Gerster, 2000). Two-color in situ hybridization was performed essentially as described by Jowett (Jowett, 1996) with minor modifications (Phillips et al., 2001).

\section{Morpholino oligomer injection}

fgf3-specific morpholino oligomer obtained from Gene Tools was diluted in Danieaux solution [ $58 \mathrm{mM} \mathrm{NaCl}, 0.7 \mathrm{mM} \mathrm{KCl}, 0.4 \mathrm{mM}$ $\mathrm{MgSO}_{4}, 0.6 \mathrm{mM} \mathrm{Ca}\left(\mathrm{NO}_{3}\right)_{2}, 5.0 \mathrm{mM}$ HEPES, $\mathrm{pH}$ 7.6] to a concentration of $5 \mu \mathrm{g} / \mu \mathrm{l}$ as previously described (Nasevicius and Ekker, 2000; Phillips et al., 2001). Approximately $1 \mathrm{nl}$ (5 ng fgf3$\mathrm{MO})$ was injected into the yolk cell at the one- to two-cell stage.

\section{Mis-expression of val}

Wild-type val was ligated into pCS2 expression vector by Andrew Waskiewicz (Cecilia Moens' laboratory) and was kindly provided as a gift. RNA was synthesized in vitro and $\sim 1 \mathrm{ng}$ of RNA was injected into the yolk of cleaving embryos at the one- to four-cell stage.

\section{RESULTS}

\section{Altered patterns of hair cells in val/val mutants}

val/val mutants produce small otic vesicles with shortened anteroposterior axes, but relatively normal dorsoventral axes. This gives the mutant ear a characteristic circular shape that is very distinct from the ovoid shape of the wild-type ear. This is thought to arise secondarily from abnormal development of the hindbrain (Moens et al., 1998), signals from which are required for normal ear development. To test this idea directly, we characterized early patterning of the otic vesicle and hindbrain in val/val mutants. In val/val mutants, the size, number and distribution of otoliths in the inner ear vary considerably (Fig. 1A,B). In wild-type embryos, otoliths form only at the anterior and posterior ends of the otic vesicle where they attach to the kinocilia of tether cells (Fig. 1C) (Riley et al., 1997). Tether 
Fig. 1. Patterns of hair cells in the otic vesicle. Lateral view of otic vesicles of live val/val (A,B) and wild-type (C) embryos viewed under DIC optics at $21 \mathrm{~h}$. val/val mutants have small, round otic vesicles, and otoliths vary in number and position. $(\mathrm{D}, \mathrm{E})$ Dorsolateral view of deltaA expression in the otic vesicle at $19 \mathrm{~h}$ in $\mathrm{val} / \mathrm{val}$ (D) and wild-type (E) embryos. Arrowheads indicate nascent tether cells. (F-H) Dorsolateral view of otic vesicles showing hair cells stained with anti-Pax2 (red) and antiacetylated tubulin (green) antibodies. (F) val/val mutant at $24 \mathrm{~h}$. Seven hair cells are distributed along the length of the anteroposterior axis of the otic vesicle. (G) val/val mutant at 30 h. An ectopic patch of hair cells (arrowhead) is evident between the anterior and posterior maculae. $(\mathrm{H})$ Wild-type embryo at 30 h. (I-K) Dorsolateral view of val/val mutants at $27 \mathrm{~h}$ stained with anti-Pax2 to visualize hair cell nuclei. The number and distribution of hair cells are variable. Anterior is towards the left in all specimens. Scale bar: $20 \mu \mathrm{m}$ in A-C; $15 \mu \mathrm{m}$ in D,E; $30 \mu \mathrm{m}$ in $\mathrm{F}-\mathrm{H} ; 40 \mu \mathrm{m}$ in $\mathrm{I}-\mathrm{K}$.

cells are the first hair cells to form and occur in pairs at both ends of the nascent otic vesicle where they facilitate localized accretion of otolith material. The supernumerary and ectopic otoliths observed in $\mathrm{val} / \mathrm{val}$ embryos were each associated with pairs of tether cells, as seen in live embryos under DIC optics (not shown). Visualizing tether cells by their expression of deltaA (dla - Zebrafish Information Network) (Haddon et al., 1998a; Riley et al., 1999) confirms that val/val mutants produce excess and ectopic tether cells (Fig. 1D). In both wild-type and val/val embryos, tether cells acquire the morphology of mature hair cells by $22 \mathrm{~h}$ (Riley et al., 1997) (data not shown) and can be visualized by nuclear staining with anti-Pax 2 antibody. This antibody was originally directed against mouse Pax2 but also binds zebrafish pax2.1 (pax2a - Zebrafish Information Network), which is preferentially expressed in maturing hair cells (Riley et al., 1999). Because of the unusual positions of some hair cells in $\mathrm{val} / \mathrm{val}$ mutants, their cell type identity was confirmed in some specimens by staining with anti-acetylated tubulin, which labels hair cell kinocilia (Haddon and Lewis, 1996). This confirmed the presence of excess and ectopic hair cells at 24 $\mathrm{h}$ in $\mathrm{val} / \mathrm{val}$ mutants (Fig. 1F). val/val mutants continue to show greater numbers of hair cells than wild-type embryos through at least $33 \mathrm{~h}$ (Fig. 2; Table 1). In addition, ectopic patches of hair cells continue to develop between the anterior and posterior maculae in most val/val mutants (Fig. 1G). However, the spatial distribution of hair cells varies widely from one specimen to the next (Fig. 1G,I-K). In general, hair cells can emerge at any position along the ventromedial surface of the otic vesicle in val/val mutants, unlike wild-type embryos in which hair cells are restricted to the anterior (utricular) and posterior (saccular) maculae. These data suggest that the

\section{Table 1}

\begin{tabular}{lccc}
\hline & \multicolumn{2}{c}{ Number of hair cells/ear at $30 \mathrm{~h}$} & \\
\cline { 2 - 3 } Genotype & Mean \pm s.d. & Range & Number \\
\hline$+/+$ & $6.9 \pm 1.1$ & $6-9$ & 28 \\
val/val & $12 \pm 1.3$ & $10-14$ & 32 \\
fgf3 $k d$ & $5.3 \pm 1.7$ & $2-8$ & 21 \\
fgf3 $k d$ in val/val & $5.7 \pm 2.4$ & $2-11$ & 33 \\
acelace & $2.9 \pm 1.0$ & $2-5$ & 19 \\
acelace; val/val & $2.5 \pm 0.7$ & $1-4$ & 28 \\
\hline
\end{tabular}

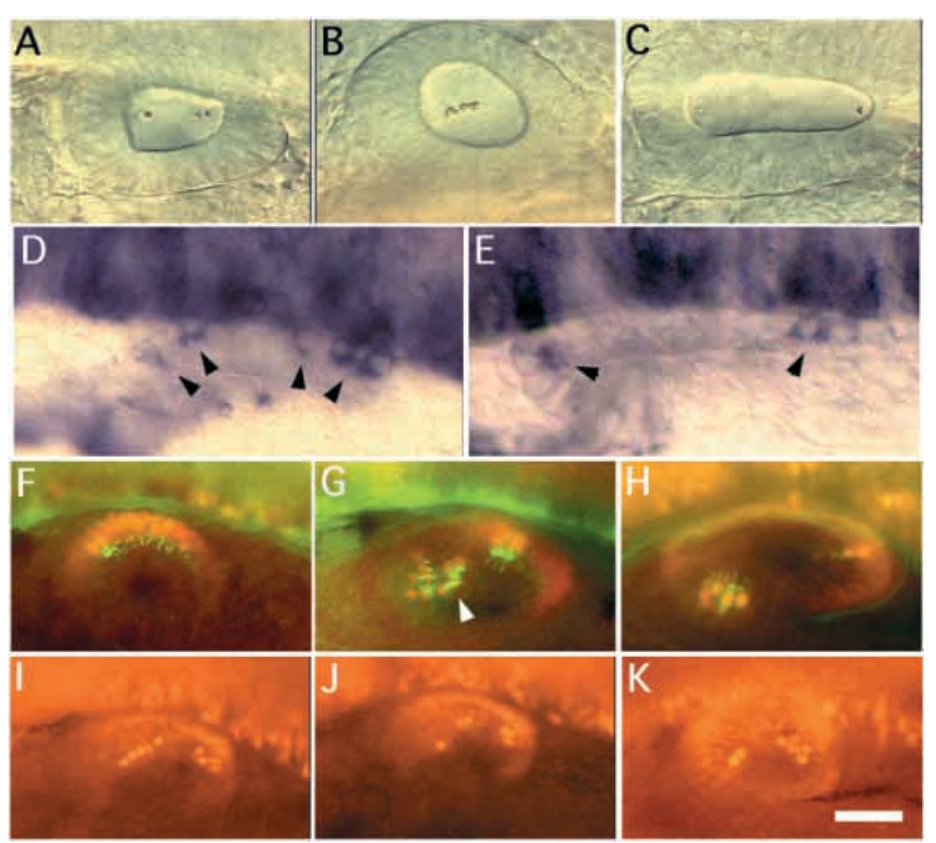

signal(s) that normally regulate the location and number of hair cells are misregulated in val/val mutants, an interpretation further supported by analysis of FGF expression in the hindbrain (see below).

\section{Altered anteroposterior patterning in val/val mutants}

We next examined expression of various otic markers to further characterize altered patterning in $\mathrm{val} / \mathrm{val}$ embryos. Expression of pax5 is first detectable in the inner ear at 17.5-18.0 h (Pfeffer et al., 1998). This expression domain is normally restricted to the anterior part of the otic vesicle adjacent to $\mathrm{r} 4$ and is

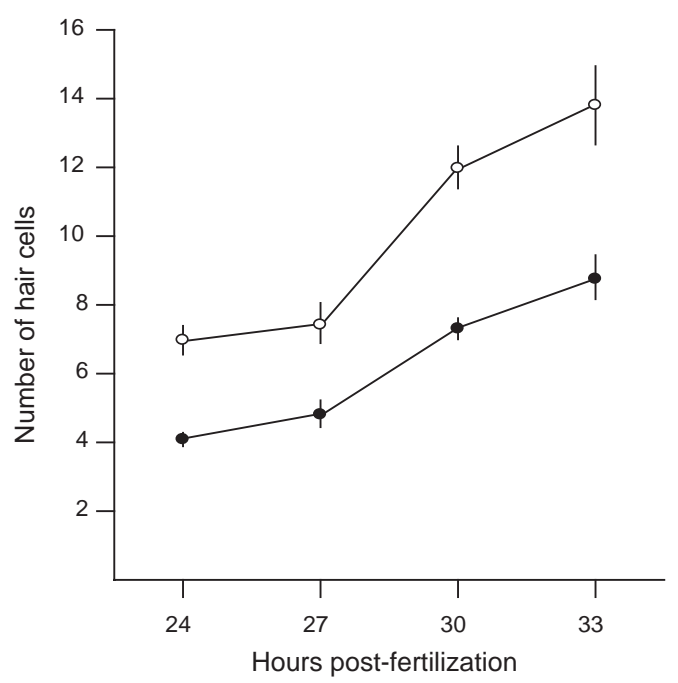

Fig. 2. Time course of hair cell formation in the otic vesicle. Embryos were fixed at the indicated times and hair cells were visualized by Pax 2 staining. Each datum is the mean number of hair cells per ear ( \pm s.d.) of 10 or more specimens. val/val mutants produce excess hair cells throughout the time course. 0 , wild type; $\bigcirc$ val/val embryos. 
maintained through at least $30 \mathrm{~h}$ (Fig. 3A,C). In val/val embryos, pax5 expression extends along the entire length of the medial wall of the otic vesicle (Fig. 3B,D). Another anterior

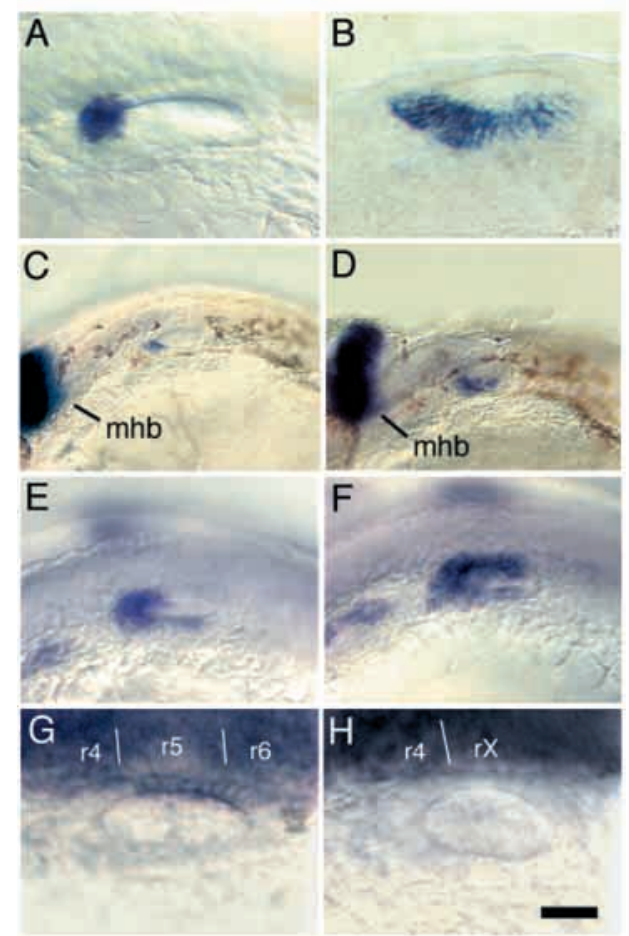

Fig. 3. Expression of AP markers in the inner ear. Lateral or dorsolateral views of the otic vesicle (anterior towards the left). (A-D) pax 5 expression at $24 \mathrm{~h}(\mathrm{~A}, \mathrm{~B})$ and $30 \mathrm{~h}$ (C,D). Staining is limited to the anterior end of the otic vesicle in wild-type embryos $(\mathrm{A}, \mathrm{C})$ but is greatly expanded in $\mathrm{val} / \mathrm{val}$ mutants $(\mathrm{B}, \mathrm{D})$. The midbrain-hindbrain border $(\mathrm{mhb})$ is indicated. (E,F) Expression of $n k x 5.1$ at $24 \mathrm{~h}$ in wild-type (E) and val/val (F) embryos. Expression is expanded posteriorly in $\mathrm{val} / \mathrm{val}$ mutants. $(\mathrm{G}, \mathrm{H})$ Expression of zp23 at $24 \mathrm{~h}$ in wild-type $(\mathrm{G})$ and $\mathrm{val} / \mathrm{val}(\mathrm{H})$ embryos. No expression is detectable in the ear in $\mathrm{val} / \mathrm{val}$ mutants. Relative positions of rhombomeres are indicated. Scale bar: $25 \mu \mathrm{m}$ in A,B, G,H; $75 \mu \mathrm{m}$ in $\mathrm{C}, \mathrm{D} ; 50 \mu \mathrm{m}$ in E,F.

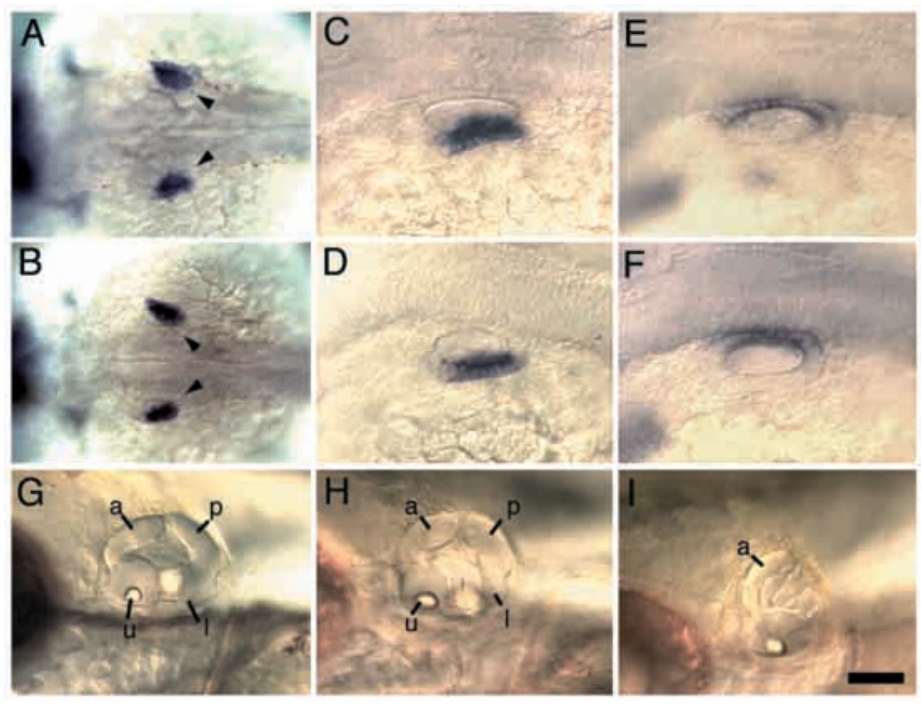

marker, $n k x 5.1$, is also expressed throughout the medial wall of the otic vesicle in val/val mutants (Fig. 3F). By contrast, $z p 23$ is normally expressed in posterior medial cells adjacent to $\mathrm{r} 5$ and $\mathrm{r} 6$ in the wild type but is not detectably expressed in val/val embryos (Fig. 3G,H). Otic patterning is not globally perturbed, however. Mutant embryos show a normal pattern of dlx3 expression in the dorsomedial epithelium (Fig. 4F). Similarly, otxl is expressed normally in ventral and lateral cells of val/val mutants (Fig. 4A-D). Based on studies in mouse, the dorsal and lateral domains of $d l x 3(d l \times 3 b-$ Zebrafish Information Network) and otxl probably help regulate development of the semicircular canals and sensory cristae (Depew at al., 1999; Krauss and Lufkin, 1999; Morsli et al., 1999; Mazan et al., 2001). It has previously been reported that formation of semicircular canals is totally disrupted in $\mathrm{val} / \mathrm{val}$ mutants (Moens et al., 1998). However, we find that this is a highly variable phenotype, ranging from grossly abnormal morphogenesis to nearly normal patterning at day 3 (Fig. 4GI). Morphology typically becomes increasingly aberrant with time, possibly resulting from improper regulation of endolymph, as seen in kreisler mutant mice (Deol, 1964; Brigande et al., 2000) (see Discussion). Regardless of whether semicircular canals develop properly, all three sensory cristae are produced and express $m s x c$ (data not shown). Thus, some aspects of axial patterning are relatively normal in val/val embryos at early stages, and the only consistent defect is that medial cells abutting the hindbrain all show anterior character. This is consistent with the hypothesis that factors locally expressed in the hindbrain regulate anterposterior fates in the medial wall of the otic vesicle, and that such factors are misregulated in the $\mathrm{rX}$ region of val/val mutants. Such misexpression could also explain the abnormal pattern of hair cells produced in val/val mutants.

\section{Expression of $f g f 3$ and $f g f 8$ in the $\mathrm{val} / \mathrm{val}$ hindbrain}

Fgf3 and Fgf8 are both expressed in the r4 anlagen during late gastrulation and cooperate to induce the otic placode (Phillips et al., 2001). We hypothesized that persistent expression of one or both of these factors in $\mathrm{r} 4$ plays a later role in patterning the otic placode and vesicle. In both wildtype and $\mathrm{val} / \mathrm{val}$ embryos, $f g f 8$ is expressed at high levels in $\mathrm{r} 4$ at $12 \mathrm{~h}$ (Fig. 5A,B) but is downregulated by $14 \mathrm{~h}$ (not shown). This argues against a role for Fgf8 in the etiology of the inner ear phenotype in val/val embryos. By contrast, $f g f 3$ expression shows a consistent difference between $\mathrm{val} / \mathrm{val}$ and wild-type embryos. In the wild type, hindbrain expression of $f g f 3$ is restricted to $\mathrm{r} 4$ and is maintained

Fig. 4. DV and ML patterning in the inner ear. (A-D) Expression of otxl at $24 \mathrm{~h}$ in wild-type $(\mathrm{A}, \mathrm{C})$ and $v a l / v a l ~(\mathrm{~B}, \mathrm{D})$ embryos. Dorsal views $(\mathrm{A}, \mathrm{B})$ show expression in the lateral epithelium of the otic vesicle (arrowheads) and lateral views (C,D) show expression in the ventral epithelium. (E,F) Dorsolateral views showing expression of $d l x 3$ at $24 \mathrm{~h}$ in wild-type (E) and val/val (F) embryos. Gene expression patterns are normal. (G-I) Lateral views of the inner ear at $72 \mathrm{~h}$ in wild-type $(\mathrm{G})$ and val/val $(\mathrm{H}, \mathrm{I})$ embryos. Morphology ranges from nearly normal to highly aberrant. Anterior is towards the left in all specimens. Abbreviations: a, anterior semicircular canal; 1, lateral semicircular canal; $p$, posterior semicircular canal; $u$, utricle. Scale bar: $100 \mu \mathrm{m}$ in A,B,G-I; $50 \mu \mathrm{m}$ in C-F. 
through at least $18 \mathrm{~h}$ when the otic vesicle forms (Fig. $5 \mathrm{C}, \mathrm{E}$, and data not shown). In val/val mutants, fgf3 shows similar developmental timing but is expressed in an expanded domain extending from $r 4$ through $r X$ (Fig. $5 \mathrm{D}, \mathrm{F})$. Within $\mathrm{rX}$, the level of expression falls off gradually towards the posterior such that there is no clear posterior limit of expression. Ectopic expression of $\mathrm{fg} 3 \mathrm{in} \mathrm{val} / \mathrm{val}$ embryos is first detectable at $10 \mathrm{~h}$, corresponding to the time when val normally begins to function in the $r 5 / 6$ anlagen (data not shown). Initially, ectopic expression of $f g f 3$ in $\mathrm{rX}$ is much weaker than in $\mathrm{r} 4$. Expression in $\mathrm{rX}$ subsequently increases to a level similar to that seen in $\mathrm{r} 4$ by $12 \mathrm{~h}$ (Fig. 5D). These data suggest that expansion of the domain of $f g f 3$ in the hindbrain could play a role in misexpression of AP markers and production of ectopic hair cells in the inner ear.

The above data also suggest that val normally functions, directly or indirectly, to exclude $f g f 3$ expression from $\mathrm{r} 5 / 6$. To explore this more fully, we examined the effects of val mis-expression by injecting val RNA into wild-type embryos. In more than half (55/98) of val-injected embryos, hindbrain expression of $f g f 3$ was dramatically reduced or ablated (Fig. 6A,B). Similar effects were seen at 10, 12 and $14 \mathrm{~h}$ (data not shown). At $24 \mathrm{~h}$, otic vesicles were usually small (15/64) or totally ablated (36/64) (Fig. 6C,D). Disrupting $f g f 3$ by itself impairs, but does not ablate, otic tissue (Phillips et al., 2001; Vendrell et al., 2001; Maroon et al., 2002). This indicates that val mis-expression affects other processes in addition to $f g f 3$ expression. Indeed, ubiquitous mis-expression of $v a l$ frequently caused truncation of the trunk and tail (46/64, Fig. 6C) and could therefore impair mesendodermal signals on which otic development relies (reviewed by Whitfield et al., 2002). However, even among embryos with normal axial development, about half showed partial loss of fgf3 expression (5/10) and impaired otic development (18/34). In many of these cases, these defects were limited to one side of the embryo (Fig. 6E,F), possibly resulting from variation in the amount of RNA inherited by early cleavage stage blastomeres. In contrast to $f g f 3$, expression of $f g f 8$ was relatively normal in most (82/85) val-injected embryos, even those with axial truncations (Fig. 6H). These data support the hypothesis that $v a l$ specifically represses $f g f 3$
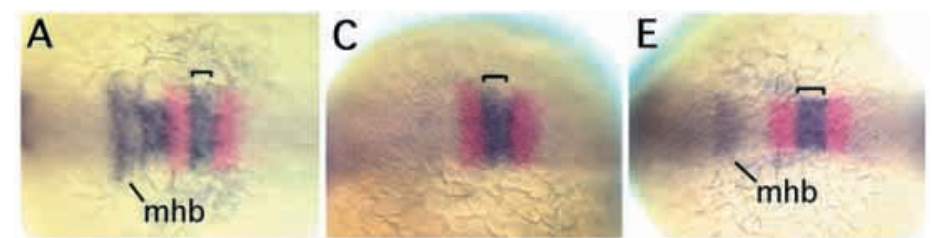

B
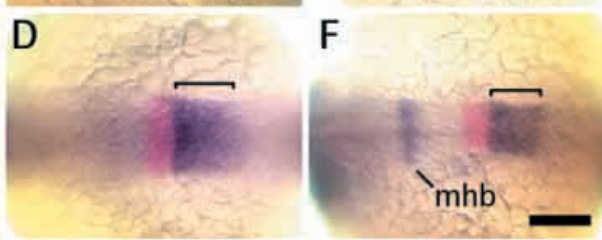

Fig. 5. Expression of $f g f 8$ and $f g f 3$ in the hindbrain. Dorsal view (anterior towards the left) of specimens double stained for Fgf gene expression (blue) and krox20 (red). Loss of krox20 staining in r5 identifies val/val mutants. (A,B) $f g f 8$ expression at $12 \mathrm{~h}$ in wild-type (A) and val/val (B) embryos. Brackets indicate the $\mathrm{r} 4$ domain of $f g f 8$. No change is detected in the mutant. (C,D) $f g f 3$ expression at $12 \mathrm{~h}$ in wild-type (C) and val/val (D) embryos. (E,F) fgf3 expression at $14 \mathrm{~h}$ in wild-type $(\mathrm{E})$ and val/val $(\mathrm{F})$ embryo. Brackets indicate the domain of $f g f 3$ corresponding to either $\mathrm{r} 4(\mathrm{C}, \mathrm{E})$ or $\mathrm{r} 4$ to $\mathrm{rX}(\mathrm{D}, \mathrm{F}) . f g f 3$ is ectopically expressed in the rX region in val/val embryos. Scale bar: $80 \mu \mathrm{m}$.

expression in the hindbrain. This is in sharp contrast to the function of the mouse homolog kreisler, which is required to activate high level expression of Fgf3 in r5 and r6 (McKay et al., 1996). Such species differences may have been important for evolutionary changes in inner ear structure and function (see Discussion).

\section{Dependence of inner ear patterning on Fgf3}

To test the role of Fgf3 in otic vesicle patterning, embryos were injected with fgf3-MO, an antisense oligomer that specifically inhibits translation of $f g f 3$ mRNA (Nasevicius and Ekker, 2000; Phillips et al., 2001; Maroon et al., 2002). Injection of fgf3-MO into wild-type embryos results in a range of defects with varying degrees of severity (Phillips et al., 2001). The size of otic vesicle is usually reduced, and about half (42/86) of Fgf3-depleted wild-type embryos show little or no pax5 expression in the inner ear (Fig. 7A). Expression of $n k x 5.1$ is

Fig. 6. Effects of mis-expressing val.

$(\mathrm{A}, \mathrm{B})$ Dorsal views showing expression of $f g f 3$ (blue) and krox20 (red) at $14 \mathrm{~h}$ in a normal embryo (A) and an embryo injected with val RNA (B). The val-injected embryo shows little or no $f g f 3$ expression in the $\mathrm{r} 4$ domain (arrowheads) and has undergone less convergence than normal. (C,D) Lateral view of a val-injected embryo at $24 \mathrm{~h}$. Trunk and tail tissues are ablated (C) and no otic vesicle is visible (D). (E,F) Dorsal views of val-injected embryos with relatively normal axial development. (E) Expression of $f g f 3$ (blue) and krox20 (red) at $14 \mathrm{~h}$. The left side of 4 shows little fef3 expression (arrowt . hindbrain border (mhb) and otic vesicles (ov). The left otic vesicle (broken circle) is severely disrupted. (G,H) Expression of $f g f 8$ at $12 \mathrm{~h}$ in a normal wild-type embryo $(\mathrm{G})$ and a val-injected embryo $(\mathrm{H})$. The val-injected embryo has a truncated axis (not shown) and has undergone less convergence than normal. Nevertheless, $f g f 8$ is expressed relatively normally in the prechordal plate (p), midbrain-hindbrain border (mhb) and rhombomere 4 (r4). Anterior is towards the left in all panels. Scale bar: $100 \mu \mathrm{m}$ in A,B,D-H; $250 \mu \mathrm{m}$ in C.
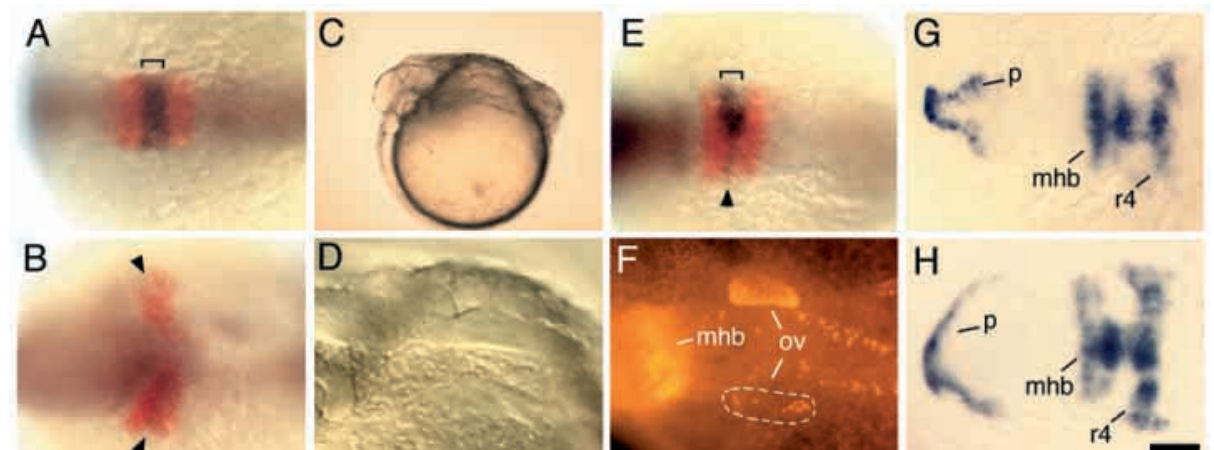
Fig. 7. Effects of $f g f 3$ knockdown on inner ear development. Dorsolateral view (anterior towards the left) of otic vesicles in embryos injected with $f g f 3-M O$. (A-C) In situ hybridization of pax5 at $24 \mathrm{~h}$ in injected wild-type (A) and injected $\mathrm{val} / \mathrm{val}(\mathrm{B}, \mathrm{C})$ embryos. Expression levels are greatly reduced in half to twothirds of embryos (see text for details). (D,E) Expression of zp23 at $24 \mathrm{~h}$ in injected wild-type (D) and injected val/val (E) embryos. Expression is detected throughout the medial wall of the otic vesicle, including cells adjacent to $\mathrm{r} 4$. (F) In situ hybridization of $n k x 5.1$ at $24 \mathrm{~h}$ in an injected val/val embryo. No expression is detected in the otic vesicle. (G-I) Anti-Pax2 staining at $30 \mathrm{~h}$ in injected wild-type $(\mathrm{G})$ and injected $\mathrm{val} / \mathrm{val}(\mathrm{H}, \mathrm{I})$ embryos. The number of hair cells is reduced relative to uninjected controls, and the majority (19/25) of val/val embryos do not produce ectopic hair cells. fgf3-depleted val/val embryos with extremely small otic vesicles (I) produced anterior hair cells only. Relative positions of rhombomeres are indicated. Scale bar: $70 \mu \mathrm{m}$ in A$\mathrm{C}, \mathrm{F} ; 50 \mu \mathrm{m}$ in $\mathrm{D}, \mathrm{E} ; 30 \mu \mathrm{m}$ in G-I.

also reduced or ablated in the otic vesicle and vestibuloacoustic ganglion in about half (30/62) of injected wild-type embryos (data not shown). By contrast, expression of zp23 often expands anteriorly in the otic vesicle to include medial cells adjacent to r4 (21/32 embryos, Fig. 7D). Hair cell production is reduced by up to $70 \%$ in severely affected embryos (Fig. 7G; Table 1, note range of data). Injection of fgf3-MO into val/val mutants leads to further reduction in the size of otic vesicle. Expression of pax 5 is strongly reduced in most cases: In one experiment, 37\% (26/71) showed pax5 expression limited to the anterior of the otic vesicle (Fig. 7B) and 38\% (27/71) showed no detectable expression (Fig. 7C). Similarly, $n k x 5.1$ is strongly reduced or ablated in about half (16/30) of injected val/val embryos (Fig. 7F). Most (12/15) val/val embryos injected with fgf3-MO express zp23 in the otic vesicle, including tissue adjacent to $\mathrm{r} 4$ (Fig. 7E). Hair cell production is reduced to a level comparable with that seen in Fgf3-depleted wild-type embryos (Table 1). In addition, depletion of Fgf3 prevents formation of ectopic hair cells in the majority (19/25) of val/val embryos (Fig. 7H,I). Thus, Fgf3-depletion prevents formation of excess and ectopic hair cells as well as misexpression of AP markers in val/val mutants. As the hindbrain is the only periotic tissue known to express $f g f 3$ at this time, we infer that the expanded domain of $f g f 3$ in $v a l / v a l$ mutants is crucial for generation of the above inner ear defects.

Fig. 8. Effects of $f g f 8$ dysfunction on inner ear development. $(A, B)$ Dorsal view of the hindbrain at $14 \mathrm{~h}$ showing expression of fgf3 (blue, with brackets) and krox20 (red) in ace/ace (A) and acelace; val/val (B) embryos. (C,D) Dorsolateral view showing pax5 expression in the otic vesicle at $24 \mathrm{~h}$ in acelace $(\mathrm{C})$ and ace/ace; val/val (D) embryos. (E,F) Dorsolateral view showing anti-Pax 2 staining in the otic vesicle at $30 \mathrm{~h}$ in ace/ace (E) and acelace; val/val (F) embryos. Relative positions of rhombomeres are indicated. Double mutants show ectopic expression of $f g f 3$ in rX (B), ectopic expression of pax5 (D) and ectopic hair cells in the otic vesicle $(\mathrm{F})$. Anterior is towards the left in all specimens. Scale bar: $80 \mu \mathrm{m}$ in $\mathrm{A}, \mathrm{B} ; 30 \mu \mathrm{m}$ in $\mathrm{C}-\mathrm{F}$.
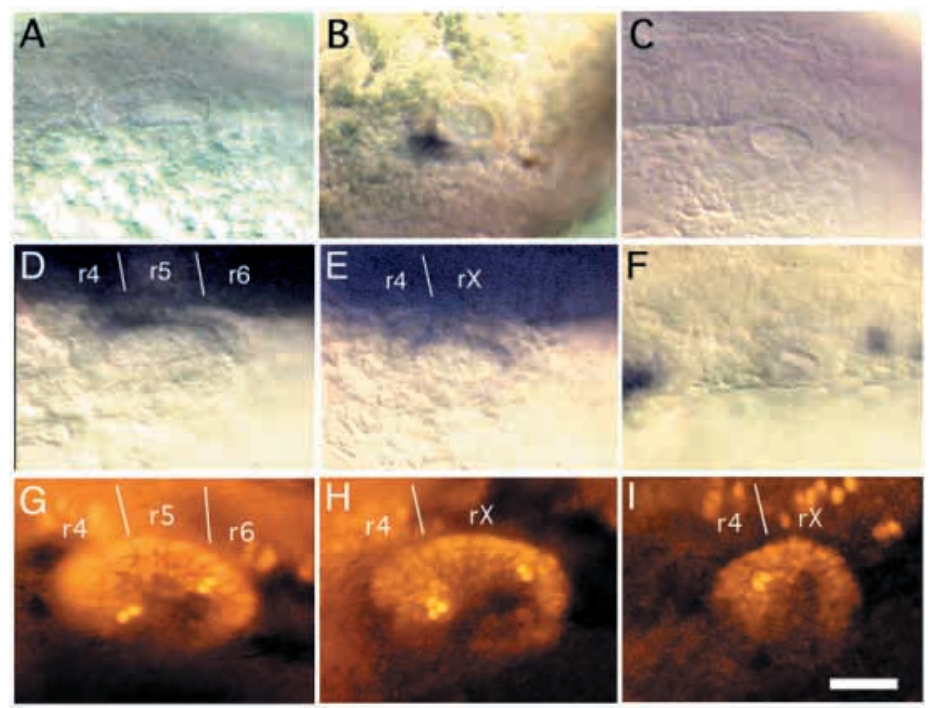

\section{Dependence of inner ear patterning on Fgf8}

Although expression of $f g f 8$ did not appear to correlate with changes in inner ear patterning in val/val mutants, we sought to characterize patterning defects in ace/ace mutants and examine genetic interactions between ace and val. Defects in ace/ace embryos are less variable than in embryos injected with fgf3-MO (Phillips et al., 2001). The otic vesicle in ace/ace mutants is reduced in size but usually retains an oval shape at $24 \mathrm{~h}$. Hair cell production is reduced by more than half in the majority of ace/ace mutants (Table 1), and more than a third (7/19) of specimens produce no posterior hair cells at all (Fig. $8 \mathrm{E})$. In ace/ace; val/val double mutants, the size of otic vesicle is further reduced and the number of hair cells is comparable with that in ace/ace single mutants (Fig. 8F; Table 1). Hair cells often form adjacent to $\mathrm{r} 4$ and/or $\mathrm{rX}$ in ace/ace; val/val double mutants and are usually located in a more medial position than are hair cells in ace/ace mutants (Fig. 8F). In addition, pax5 is expressed along the full length of the anteroposterior axis of the ear (Fig. 8D). Expression of $n k x 5.1$ is also expanded in acelace-val/val double mutants, while zp23 is not expressed (data not shown). Thus, the ace mutation strongly perturbs inner ear patterning, but loss of $f g f 8$ function does not suppress the patterning defects associated with the val mutation. This is probably because expression of $f g f 3$ is expanded in the hindbrain of ace/ace; val/val double mutants as in val/val mutants (Fig. 8B). Together, these data indicate that val and
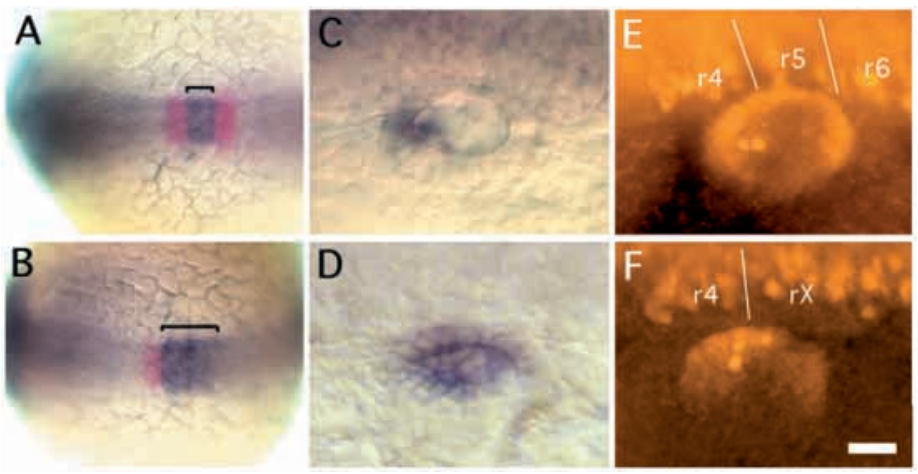
ace affect different developmental pathways, and that the early patterning defects seen in the val/val mutant ear are not caused by mis-regulation of $f g f 8$ expression.

\section{DISCUSSION}

\section{Fgf3, Fgf8 and hindbrain signaling}

Development of the first hair cells is normally restricted to regions of the otic placode directly adjacent to r4 and r6 (Fig. 1), suggesting that signals emitted by those rhombomeres specify the equivalence groups from which hair cells emerge. Data presented here suggest that Fgf3 is an important r4derived factor that regulates formation of anterior hair cells, as well as expression of various AP markers in the ear. In val/val embryos, $f g f 3$ is expressed ectopically in rX (Fig. 5), and ectopic hair cells form within the adjacent otic vesicle (Fig. 1). Expression of $n k x 5.1$ and pax5, which are normally restricted to the anterior region of the placode next to r4, expand posteriorly in $\mathrm{val} / \mathrm{val}$ mutants to include all cells abutting the hindbrain (Fig. 3). The posterior marker zp23 is not expressed in the otic vesicle in val/val mutants. Depletion of Fgf3 suppresses all of the above patterning defects in the val/val mutant ear. Moreover, in many Fgf3-depleted embryos, anterior otic markers are totally ablated and zp23 expression expands anteriorly to include cells adjacent to $\mathrm{r} 4$.

The fact that any hair cells are produced at all in Fgf3depleted embryos indicates that additional hair cell-inducing factors must be present. $f g f 8$ is clearly required for normal hair cell formation and could partially compensate for loss of $f g f 3$ (Reifers et al., 1998; Phillips et al., 2001). However, several observations indicate that the role of $f g f 8$ is distinct from that of $f g f 3$. First, periotic expression of $f g f 8$ declines sharply just before the placode forms at $14 \mathrm{~h}$, thereby limiting its ability to influence later otic patterning. Second, expression patterns of $n k x 5.1$, pax 5 and zp23 are not altered in acelace embryos (Fig. 8C, and data not shown), indicating that AP patterning is relatively normal. Third, loss of $f g f 8$ inhibits hair cell formation but does not prevent formation of ectopic hair cells in val/val mutants. The latter are dependent on $f g f 3$ instead. Thus, in contrast to $f g f 3$, there is little evidence to suggest that the $\mathrm{r} 4$ domain of $f g f 8$ regulates regional patterning in the otic placode. Instead, $f g f 8$ may play a more general role in stimulating hair cell competence during the process of placode induction.

Paradoxically, anterior hair cells are not as severely impaired in ace/ace mutants as are posterior hair cells. Posterior hair cells are totally ablated in about $1 / 3$ of ace/ace mutants. This is difficult to explain based solely on the expression domain of $f g f 8$, but may reflect changes in the dimensions of the otic placode. In ace/ace mutants, the otic placode is often reduced to a domain juxtaposed to $\mathrm{r} 4$ and $\mathrm{r} 5$ only. Thus, secretion of Fgf3 from r4 may be sufficient to induce some anterior hair cells in the absence of Fgf8, whereas cells in the posterior otic placode may lie too far from r6 to benefit from inductive factors possibly secreted from there. No clear candidates for r6-specifc inducers are known, but the Fgf-inducible genes erm, pea3 and sprouty4 are expressed in r6 (Fürthauer et al., 2001; Raible and Brand, 2001; Roehl and Nüsslein-Volhard, 2001) (S.-J. K., B. T. P., R. H. and B. B. R., unpublished), suggesting that at least one as yet unidentified Fgf homolog is expressed there.
The reason for expanded expression of $f g f 3$ in $\mathrm{val} / \mathrm{val}$ mutants is not clear, but there are several possibilities. First, this could result from mis-specification of segment identity in the rX territory. Several other genes normally expressed in adjacent segments, including hoxbl in $\mathrm{r} 4$ and hoxb4 in $\mathrm{r} 7$, eventually come to be expressed in rX (Prince et al., 1998). However, these changes do not occur until 20 somites $(19 \mathrm{~h})$. By contrast, expression of $f g f 3$ in $\mathrm{rX}$ is first detected at $10 \mathrm{~h}$ in val/val mutants, corresponding to the time when val normally begins to function (Moens et al., 1998). This raises the alternative possibility that Val protein normally acts to transcriptionally repress $f g f 3$. In support of this, mis-expression of $v a l$ inhibits r4-expression of $f g f 3$, but not $f g f 8$ (Fig. 6). Direct support for transcriptional regulation by Val will require analysis of the promoter/enhancer regions of $f g f 3$.

\section{Comparison of val and kreisler}

In sharp contrast to val function in zebrafish, mouse kreisler is required, directly or indirectly, for upregulation of Fgf3 in r5 and r6 (McKay et al., 1996). This difference is notable because so many other aspects of early hindbrain and ear development are conserved between these species. The high degree of sequence identity leaves little doubt that the zebrafish genes are orthologous to kreisler and Fgf3 (Kiefer et al., 1996a; Moens et al., 1998). There are, however, differences in the $\mathrm{N}$ - and $\mathrm{C}$ terminal regions of Fgf3 in zebrafish and mouse. These regions are thought to be important for mediating the characteristic receptor binding preferences and signaling properties of Fgf3. Nevertheless, these functional properties are actually very similar between the fish and mouse proteins (Kiefer et al., 1996b). This, combined with the broad similarities in their expression patterns and involvement in early otic development, strengthen the notion that the fish and mouse $f g f 3$ genes are indeed orthologs. Because zebrafish often has multiple homologs of specific tetrapod genes, it is possible that a second fgf3 gene might be present in the zebrafish genome that shows an expression pattern more like the mouse gene. If so, it will be important to address its function as well. However, we have shown that the known $f g f 3$ ortholog plays an essential role in the etiology of the ear phenotype in val/val embryos, as key aspects of the phenotype are suppressed by injecting fgf3-MO. Morpholino oligomers are highly gene-specific in their effects, and even though they do not totally eliminate gene function, they generate phenotypes that are indistinguishable from those caused by known null mutations (Nasevicius and Ekker, 2000; Phillips et al., 2001; Raible and Brand, 2001; Maroon et al., 2002). On balance, it appears that the general role of Fgf3 in otic development has been conserved in mouse and fish but that differential regulation in the hindbrain represents a real difference between these species.

Considering the above differences in hindbrain signaling, one might expect the ear phenotypes in val/val and Mafb/Mafb mutants to be quite different. Instead, the phenotypes appear strikingly similar. In Mafb/Mafb embryos, as in val/val embryos, development of the otic vesicle is highly variable and defects can be seen in virtually all regions of the labyrinth (Deol, 1964). In Mafb/Mafb mutants, formation of the wall of the otic capsule is often incomplete, with large gaps through with membranous epithelia protrude, and morphology of the labyrinth is usually grossly abnormal. Such global disruption may be related to buildup of excess fluid pressure due to failure 
of the endolymphatic duct to form in many or most Mafb/Mafb mutants (Deol, 1964; Brigande et al., 2000). Whether a similar problem occurs in val/val mutants is not clear. The existence of an endolymphatic duct in zebrafish has only recently been documented (Bever and Fekete, 2002), but it does not begin to form until around day 8 . Most val/val mutants die before this time, and they often begin to show defects in morphogenesis (e.g. of the semicircular canals) by $72 \mathrm{~h}$ (Fig. 4, and data not shown). Although these early defects cannot be explained by the absence of an endolymphatic duct, mutant ears often appear swollen and distended by day 3, suggesting a buildup of endolymphatic pressure. It is possible that cellular functions normally required to maintain a proper fluid balance in the early vesicle are mis-regulated in val/val mutants. Thus, hydrops may be an important contributing factor to the defects in both Mafb/Mafb and val/val mutants.

Another similarity between Mafb/Mafb and val/val mutants is that they both form ectopic patches of hair cells. However, this phenotype has a completely different etiology in the two species. In tetrapod vertebrates, sensory epithelia do not begin to differentiate until after the various chambers of the labyrinth begin to form. Thus, formation of ectopic hair cells in $M a f b / M a f b$ mutants probably reflects the general disorganization of, and chaotic protrusions from, the labyrinth (Deol, 1964). By contrast, sensory epithelia in zebrafish begin to differentiate much earlier. Macular equivalence groups are already specified at $14 \mathrm{~h}$ when the placode first forms (Haddon et al., 1998a; Whitfield et al., 2002), and the first hair cells (visualized by the presence of kinocilia) are evident as soon as the lumen of the vesicle forms at $18.5 \mathrm{~h}$ (Riley et al., 1997). Thus, formation of ectopic hair cells in val/val mutants reflects an early defect in cell fate specification rather than a later defect in morphogenesis. It is noteworthy that there have been no detailed molecular studies of otic development in Mafb/Mafb mutants, so a direct comparison of early pattern formation is not yet possible.

\section{Evolutionary implications}

It is interesting to consider that the altered pattern of $f g f 3$ expression in the val/val mutant hindbrain closely resembles the normal pattern of $\mathrm{Fgf3}$ expression in chick and mouse embryos (Mahmood, 1995; Mahmood, 1996; McKay et al., 1996). Analysis of val/val mutants suggests that misexpression of $f g f 3$ in $\mathrm{rX}$ leads to development of excess and ectopic hair cells in the otic vesicle. It is possible that evolutionary changes that led to normal expression of $F g f 3$ in r5/6 in amniotes were crucial for evolution of the cochlea, which has no known counterpart in anamniote vertebrates (Lewis et al., 1985). In the mouse, development of the cochlea requires FGF signaling at early otic vesicle stages (Pirvola et al., 2000). The FGF receptor isoform FGFR-2(IIIb) is expressed in the otic epithelium juxtaposed to the hindbrain. Targeted disruption of this isoform leads to severe dysgenesis of the cochlea. Cochlear development is also impaired in Fgf3-null and Mafb/Mafb mutant mice (Deol, 1964; Mansour et al., 1993). In Xenopus, Fgf3 expression shows a pattern intermediate between that of zebrafish and amniotes: The frog gene is initially expressed in r3 through r5 and only later becomes restricted to r4 (Lombardo et al., 1998). Although amphibians do not possess a cochlea, they do show modifications of the posterior otic vesicle that give rise to the basilar and amphibian papillae, auditory organs not found in fish (reviewed by Lewis et al., 1985). Thus, expression of $f g f 3$ in more posterior regions of the hindbrain correlates with elaborations of the inner ear that may have been essential for enhancing auditory function in terrestrial environments.

This work was supported by National Institutes of Health, NIDCD grant 5 R01 DC03806. We thank Andrew Waskiewicz and Cecilia Moens for their kindness and generosity in providing the pCS2-val expression vector, and Arne Lekven for helpful discussion of the data. We also thank Peter Pfeffer, Eva Bober and Giselbert Hauptmann for providing pax5, nkx5.1 and zp23 cDNAs.

\section{REFERENCES}

Adamska, M., Leger, S., Brand, M., Hadrys, T., Braun, T. and Bober, E. (2000). Inner ear and lateral line expression of a zebrafish $N k x 5-1$ gene and its downregulation in the ears of FGF8 mutant, ace. Mech. Dev. 97, 161165.

Appel, B. and Eisen, J. S. (1998). Regulation of neuronal specification in the zebrafish spinal cord by Delta function. Development 125, 371-380.

Bever, M. M. and Fekete, D. M. (2002). Atlas of the developing inner ear in zebrafish. Dev. Dyn. 223, 536-543.

Brand, M., Heisenberg, C.-P., Jiang, Y.-L., Beuchle, D., Lun, K., FurutaniSeiki, M., Granato, M., Hafter, P., Hammerschmidt, M., Kane, D. et al. (1996). Mutations in zebrafish genes affecting the formation of the boundary between the midbrain and hindbrain. Development 123, 179-190.

Brigande, J. V., Keirnan, A. E., Gao, X., Iten, L. E. and Fekete, D. M. (2000). Molecular genetics of pattern formation in the inner ear: Do compartment boundaries play a role? Proc. Natl. Acad. Sci. USA 97, 1170011706.

Cordes, S. P. and Barsh, G. S. (1994). The mouse segmentation gene $k r$ encodes a novel basic domain-leucine zipper transcription factor. Cell 79, 1025-1034.

Deol, M. S. (1964). The abnormalities of the inner ear in kreisler mice. $J$. Embryol. Exp. Morphol. 12, 475-490.

Depew, M. J., Liu, J. K., Long, J. E., Presley, R., Meneses, J. J., Pedersen, R. A. and Rubenstein, J. L. R. (1999). Dlx5 regulates regional development of the branchial arches and sensory capsules. Development 126, 3831-3846.

Ekker, M., Akimenko, M.-A., Bremiller, R. and Westerfield, M. (1992). Regional expression of three homeobox transcripts in the inner ear of zebrafish embryos. Neuron 9, 27-35.

Fürthauer, M., Reifers, F., Brand, M., Thisse, B. and Thisse, C. (2001) sprouty 4 acts in vivo as a feedback-induced antagonist of FGF signalling in zebrafish. Development 128, 2175-2186.

Gallagher, B. C., Henry, J. J. and Grainger, R. M. (1996). Inductive processes leading to inner ear formation during Xenopus development. Dev. Biol. 175, 95-107.

Groves, A. K. and Bronner-Fraser, M. (2000). Competence, specification and commitment in otic placode induction. Development 127, 3489-3499.

Haddon, C. and Lewis, J. (1996). Early ear development in the embryo of the zebrafish, Danio rerio. J. Comp. Neurol. 365, 113-128.

Haddon, C., Jiang, Y.-L., Smithers, L. and Lewis, J. (1998a). DeltaNotch signalling and the patterning of sensory cell differentiation in the zebrafish ear: evidence from the mind bomb mutant. Development 125, 4637-4644.

Haddon, C., Smithers, L., Schneider-Maunoury, S., Coche, T., Henrique, D. and Lewis, J. (1998b). Multiple delta genes and lateral inhibition in zebrafish primary neurogenesis. Development 125, 359-370.

Harrison, R. G. (1935). Factors concerned in the development of the ear in Ablystoma punctatum. Anat. Rec. 64 Suppl., 38-39.

Hauptmann, G. and Gerster, T. (2000). Combined expression of zebrafish Brn-1- and Brn-2-related POU genes in the embryonic brain, pronephric primordium, and pharyngeal arches. Dev. Dyn. 218, 345-358.

Hutson, M. R., Lewis, J. E., Nguyen-Luu, D., Lindberg, K. H. and Barald, K. F. (1999). Expression of Pax 2 and patterning of the chick inner ear. $J$. Neurocytol. 28, 795-807.

Jacobson, A. G. (1963). The determination and positioning of the nose, lens, and ear. I. Interactions within the ectoderm, and between the ectoderm and underlying tissue. J. Exp. Zool. 154, 273-283. 
Jowett, T. (1996). Double fluorescent in situ hybridization to zebrafish embryos. Trends Genet. 12, 387-389.

Kiefer, P., Strahle, U. and Dickson, C. (1996a). The zebrafish Fgf-3 gene: cDNA sequence, transcript structure and genomic organization. Gene 168, 211-215.

Kiefer, P., Mathieu, M., Mason, I. and Dickson, C. (1996b). Secretion and mitogenic activity of zebrafish FGF3 reveal intermediate properties relative to mouse and Xenopus homologues. Oncogene 12, 1503-1511.

Krauss, P. and Lufkin, T. (1999). Mammalian Dlx homeobox gene control of craniofacial and inner ear morphogenesis. J. Cell. Biochem. Suppl. 32/33, 133-140.

Ladher, R. K., Anakwe, K. U., Gurney, A. L., Schoenwolf, G. C. and Francis-West, A. L. (2000). Identification of synergistic signals initiating inner ear development. Science 290, 1965-1967.

Lewis, E. R., Leverenz, E. L. and Bialek, W. S. (1985). The Vertebrate Inner Ear. Boca Raton, FL: CRC Press.

Li, Y., Allende, M. L., Finkelstein, R. and Weinberg, E. S. (1994). Expression of two zebrafish orthodenticle-related genes in the embryonic brain. Mech. Dev. 48, 229-244.

Lombardo, A. and Slack, J. M. W. (1998). Postgastrulation effects of fibroblast growth factor on Xenopus development. Dev. Dyn. 212, 75-85.

Lombardo, A., Isaacs, H. V. and Slack, J. M. W. (1998). Expression and functions of FGF-3 in Xenopus development. Int. J. Dev. Biol. 42, 11011107.

Mahmood, R., Kiefer, P., Guthrie, S., Dickson, C. and Mason, I. (1995). Multiple roles for FGF-3 during cranial neural development in the chicken. Development 121, 1399-1410.

Mahmood, R., Mason, I. J. and Morrisskay, G. M. (1996). Expression of $F G F-3$ in relation to hindbrain segmentation, otic pit position and pharyngeal arch morphology in normal and retinoic acid exposed mouse embryos. Anat. Embryol. 194, 13-22.

Mansour, S. L., Goddard, J. M. and Capecchi, M. R. (1993). Mice homozygous for a targeted disruption of the proto-oncogene int-2 have developmental defects in the tail and inner ear. Development 117, 13-28.

Maroon, H., Walshe, J., Mahmood, R., Kiefer, P., Dickson, C. and Mason, I. (2002). Fgf3 and Fgf8 are required together for formation of the otic placode and vesicle. Development 129, 2099-2108.

Mazan, S., Jailard, D., Baratte, B. and Janvier, P. (2001). Otxl genecontrolled morphogenesis of the horizontal semicircular canal and the origin of the gnathostome characteristics. Evol. Dev. 2, 186-193.

McKay, I. J., Lewis, J. and Lumsden, A. (1996). The role of FGF-3 in early inner ear development: an analysis in normal and kreisler mutant mice. Dev. Biol. 174, 370-378.

Mendonsa, E. S. and Riley, B. B. (1999). Genetic analysis of tissue interactions required for otic placode induction in the zebrafish. Dev. Biol. 206, 100-112.

Moens, C. B., Yan, Y.-L., Appel, B., Force, A. G. and Kimmel, C. B. (1996). valentino: a zebrafish gene required for normal hindbrain segmentation. Development 122, 3981-3990.

Moens, C. B., Cordes, S. P., Giorgianni, M. W., Barsh, G. S. and Kimmel, C. B. (1998). Equivalence in the genetic control of hindbrain segmentation in fish and mouse. Development 125, 381-391.

Morsli, H., Tuorto, F., Choo, D., Postiglione, M. P., Simeone, A. and Wu, D. K. (1999). Otxl and Otx2 activities are required for the normal development of the mouse inner ear. Development Suppl., 2333-2343.
Nasevicius, A. and Ekker, S. C. (2000). Effective targeted gene 'knockdown' in zebrafish. Nat. Genet. 26, 216-220.

Pfeffer, P. L., Gerster, T., Lun, K., Brand, M. and Büsslinger, M. (1998). Characterization of three novel members of the zebrafish Pax2/5/8 family: dependency of Pax5 and Pax8 expression on the Pax2.1 (noi) function. Development 125, 3063-3074.

Phillips, B. T., Bolding, K. and Riley, B. B. (2001). Zebrafish $f g f 3$ and $f g f 8$ encode redundant functions required for otic placode induction. Dev. Biol. 235, 351-365.

Pirvola, U., Spencer-Dene, B., Xing-Qun, L., Kettunen, P., Thesleff, I., Fritzsch, B. and Dickson, C. (2000). FGF/FGFR-2 (IIIb) signaling is essential for inner ear morphogenesis. J. Neurosci. 20, 6125-6134.

Prince, V. E., Moens, C. B., Kimmel, C. B. and Ho, R. K. (1998). Zebrafish hox genes: expression in the hindbrain region of the wild-type and mutants of the segmentation gene, valentino. Development 125, 393-406.

Raible, F. and Brand, M. (2001). Tight transcriptional control of the ETS domain factors Erm and Pea3 by Fgf signaling during early zebrafish development. Mech. Dev. 107, 105-117.

Reifers, F., Bohli, H., Walsh, E. C., Crossley, P. H. and Stainier, D. Y. R. (1998). Fgf8 is mutated in zebrafish acerebellar (ace) mutants and is required for maintenance of midbrain-hindbrain boundary and somitogenesis. Development 125, 2381-2395.

Represa, J., Leon, Y., Miner, C. and Giraldez, F. (1991). The int-2 protooncogene is responsible for induction of the inner ear. Nature 353, 561563.

Riley, B. B., Zhu, C., Janetopoulos, C. and Aufderheide, K. J. (1997). A critical period of ear development controlled by distinct populations of ciliated cells in the zebrafish. Dev. Biol. 191, 191-201.

Riley, B. B., Chiang, M.-Y., Farmer, L. and Heck, R. (1999). The deltaA gene of zebrafish mediates lateral inhibition of hair cells in the inner ear and is regulated by pax2.1. Development 126, 5669-5678.

Roehl, H. and Nüsslein-Volhard, C. (2001). Zebrafish pea3 and erm are general targets of FGF8 signalling. Curr. Biol. 11, 503-507.

Stachel, S. E., Grunwald, D. J. and Meyers, P. Z. (1993). Lithium perturbations and goosecoid expression identify a dorsal specification pathway in the pregastrula zebrafish. Development 117, 1261-1274.

Stone, L. S. (1931). Induction of the ear by the medulla and its relation to experiments on the lateralis system in amphibia. Science 74, 577.

Vendrell, V., Carnicero, E., Giraldez, F., Alonso, M. T. and Schimmang, T. (2000). Induction of inner ear fate by FGF3. Development 127, 155165.

Vendrell, V., Gimnopoulos, D., Beckler, T. and Schimmang, T. (2001). Functional analysis of FGF3 during zebrafish inner ear development. Int. J. Dev. Biol. 45, S105-S106.

Waddington, C. H. (1937). The determination of the auditory placode in the chick. J. Exp. Biol. 14, 232-239.

Whitfield, T. T., Riley, B. B., Chiang, M.-Y. and Phillips, B. (2002). Development of the zebrafish inner ear. Dev. Dyn. 223, 427-458.

Woo, K. and Fraser, S. E. (1998). Specification of the hindbrain fate in the zebrafish. Dev. Biol. 197, 283-296.

Wu, D. K., Nunes, F. D. and Choo, D. (1998). Axial specification for sensory organs versus non-sensory structures of the chicken inner ear. Development 125, 11-20.

Yntema, C. L. (1933). Experiments on the determination of the ear ectoderm in the embryo of Ablystoma punctatum. J. Exp. Zool. 65, 317-352. 